\title{
Wasf2: A novel target of intermittent parathyroid hormone administration
}

\author{
MAKI UYAMA $^{1,2}$, MASAMITSU KAWANAMI ${ }^{2}$ and MASATO TAMURA ${ }^{1}$ \\ Departments of ${ }^{1}$ Biochemistry and Molecular Biology, ${ }^{2}$ Periodontology and Endodontology, \\ Graduate School of Dental Medicine, Hokkaido University, Sapporo 060-8586, Japan
}

Received December 20, 2012; Accepted February 21, 2013

DOI: $10.3892 / \mathrm{ijmm} .2013 .1315$

\begin{abstract}
Systemic intermittent administration of parathyroid hormone (PTH) stimulates bone formation in animals and humans, and recombinant human PTH1-34 (teriparatide) is used clinically for the treatment of osteoporosis. In this study, we investigated the regulation of gene expression by intermittent PTH administration in MC3T3-E1 osteoblastic cells. We found that intermittent PTH1-34 administration downregulated Wiskott-Aldrich syndrome protein family member (Wasf) 2 mRNA expression. Wnt inhibitor, IWP-2, and protein kinase $\mathrm{C}$ inhibitor, Go6976, inhibited this downregulation. However, continuous PTH did not affect Wasf2 expression. Transfection of Wasf 2 siRNA reduced bone sialoprotein (BSP) mRNA expression in a similar manner following intermittent PTH administration in MC3T3-E1 cells. These results identify Wasf 2 as a novel target of intermittent PTH administration via the Wnt and phosphoinositide-dependent protein kinase signaling pathways, and the resulting regulation of BSP expression may contribute to the anabolic effects of PTH.
\end{abstract}

\section{Introduction}

Human parathyroid hormone (PTH), an 84-amino acid peptide (PTH 1-84), is a principal hormone that regulates bone remodeling via its actions on both bone formation and resorption. Osteoblast lineage cells are the target cells for the effects of PTH on bone tissue. When administered intermittently by daily subcutaneous injection, recombinant human PTH1-34 (teriparatide) increases bone mineral density and reduces the incidence of skeletal fractures in osteoporosis ('anabolic' effects of PTH) (1). In contrast, the 'catabolic' effects result from pathological conditions in which the parathyroid glands

Correspondence to: Professor Masato Tamura, Department of Biochemistry and Molecular Biology, Graduate School of Dental Medicine, Hokkaido University, North 13, West 7, Sapporo 060-8586, Japan

E-mail: mtamura@den.hokudai.ac.jp

Key words: parathyroid hormone, osteoblasts, Wasf2 continuously secrete an excess of hormone. Such continuous secretion of PTH induces bone resorption by stimulation of receptor activator of nuclear factor (NF)- $\mathrm{B}$ ligand (RANKL) and inhibition of osteoprotegerin (OPG) in osteoblasts (2). The effects of PTH on osteoblasts are known to be mediated via binding of PTH to the seven membrane-spanning $G$ protein-coupled receptor, $\mathrm{PTH}$ receptor 1 (PTHR1), and activation of both the cyclic AMP (cAMP)-dependent protein kinase A (PKA) pathway and the phosphoinositide-dependent protein kinase $\mathrm{C}$ (PKC) pathway (3). Intermittent PTH administration in mice has been shown to increase osteoblast numbers by stimulating survival signaling, preventing their apoptosis, and thus increasing bone formation (4). Intermittent PTH also affects the synthesis of many osteogenic growth factors and cytokines, as well as that of their antagonists (1). Insulin-like growth factor (IGF)-1 and fibroblast growth factor (FGF)-2 may contribute to the anabolic effect of intermittent PTH in increasing osteoblast numbers (1). Intermittent PTH also promotes osteoblast differentiation, activating Wnt signaling in osteoblasts, and inhibiting the Wnt antagonist, sclerostin, in osteocytes (5). However, the molecular and cellular mechanisms underlying the anabolic action of PTH are not completely understood and remain controversial.

The Wiskott-Aldrich syndrome protein (WASP) is a cytoplasmic protein implicated in regulating the actin cytoskeleton and cytoskeletal reorganization involved in cellular functions such as migration, phagocytosis and immune synapse formation (6). WASP family protein member 2 (Wasf2; also known as WASP family verprolin-homologous protein 2; WAVE2) is one of the WASP family proteins which is ubiquitously expressed in mammals (7). Yamazaki et al (8) demonstrated that Wasf2 is crucial for Rac-induced membrane ruffling, which is important in cell motility. Wasf2-deficient mice survived only until embryonic day 12.5 and displayed growth retardation and certain morphological defects (9). Since remodeling of the cytoskeleton is involved in mechanotransduction, Wasf 2 is implicated in this process. To date, however, little is known concerning the regulation and molecular basis of action of Wasf 2 in osteoblasts.

In this study, we investigated the regulation of gene expression following intermittent PTH administration in osteoblastic MC3T3-E1 cells. Here, we showed that intermittent PTH regulated Wasf 2 expression and that the Wnt inhibitor, IWP-2, or the protein kinase C inhibitor, Go6976, inhibited 
this downregulation, indicating that Wasf 2 is a novel target of intermittent PTH administration via the Wnt and PKC signaling pathways.

\section{Materials and methods}

Cell cultures. Cells of the mouse cell line MC3T3-E1 were cultured in $\alpha$-MEM containing $100 \mu \mathrm{g} / \mathrm{ml}$ kanamycin (Meiji, Tokyo, Japan) and supplemented with $10 \%$ fetal bovine serum (FBS; SAFC Bioscience, Inc., Lenexa, KS, USA) at $37^{\circ} \mathrm{C}$ in 100-mm cell culture dishes (Corning Inc., Corning, NY, USA) in a humidified atmosphere of $5 \% \mathrm{CO}_{2}$ in air.

Compounds and reagents. PTH1-34, PTH3-34, Go6976, IWR-1 and dorsomorphin were purchased from SigmaAldrich (St. Louis, MO, USA). IWP-2 was obtained from Stemgent (San Diego, CA, USA). The Wasf2 siRNA and silencer negative control scramble siRNA (no. 4611; Ambion) were purchased from Applied Biosystems (Foster City, CA, USA). Recombinant human bone morphogenetic protein (BMP) 2 was kindly supplied by Astellas Pharma Inc. (Tokyo, Japan).

PTH administration. MC3T3-E1 cells were plated at $1 \times 10^{5}$ cells $/ \mathrm{cm}^{2}$. After $24 \mathrm{~h}$, the cells were cultured in the presence of $10^{-8} \mathrm{M}$ PTH or in control medium for 1, 6 (intermittent) or $48 \mathrm{~h}$ (continuous) within each 48 -h incubation cycle. These cycles were repeated three times. The cells were harvested after 6 days.

Microarray analysis. Total RNA was extracted from the cells using the ReliaPrep ${ }^{\text {TM }}$ RNA Cell Miniprep System (Promega), and quality was evaluated by Bioanalyzer (Agilent Technologies, Palo Alto, CA, USA). Total RNA was labeled with Cy3. Samples were hybridized using a SurePrint G3 Mouse GE microarray kit (Agilent Technologies) according to the manufacturer's protocol. Arrays were scanned with a G2539A Microarray Scanner System. Data were analyzed using GeneSpringer GX software (both from Agilent Technologies).

Reverse transcription-polymerase chain reaction (RT-PCR). Total RNA was extracted from the cells at the indicated time points using Isogen (Nippongene, Toyama, Japan), and RT-PCR was performed as previously described (10). The primer sequences for each gene were as follows: Wasf2, 5-TTGCCA AAGCCCTCATAAAC-3 (forward) and 5-AGCCAGGGTA CCATCAACAG-3 (reverse); OPG, 5-TCCTGGCACCTACC TAAAACAGCA-3 (forward) and 5-CTACACTCTCGGCATT CACTTTGG-3 (reverse); RANKL, 5-GTCACTCTGTCCTC TTGGTAC-3 (forward) and 5-TGAAACCCCAAAGTACG TCG-3 (reverse); osterix, 5-GGGTTAAGGGGAGCAAAGTC AGAT-3 (forward) and 5-CTGGGGAAAGGAGGCACAAAG AAG-3 (reverse); glyceraldehyde-3-phosphate dehydrogenase (GAPDH), 5'-TCCACCACCCTGTTGCTGTA-3' (forward) and 5'-ACCACAGTCCATGCCATCAC-3' (reverse).

To account for any difference in the amount of RNA, GAPDH was chosen as our endogenous control and amplified using the primers described above. The amplification products were electrophoresed on $2 \%$ agarose gels.
Quantification of gene expression by quantitative reverse transcription-polymerase chain reaction $(q R T-P C R)$. qRT-PCR was performed using Assay-on-Demand ${ }^{\mathrm{TM}}$ TaqMan probes (Applied Biosystems) and the StepOne ${ }^{\circledR}$ real-time PCR system. The relative level of gene expression was quantified using the comparative $\mathrm{C}_{\mathrm{T}}$ method with $G A P D H$ as the endogenous control.

Transfection of small interfering RNA (siRNA). The Wasf2 siRNA sequences for each gene were as follows: 5'-CGUA AAAUCAAGACACGCAtt-3' (sense) and 5'-UGCGUGUCU UGAUUUUACGtg-3' (antisense). MC3T3-E1 cells were plated at $1 \times 10^{5}$ cells $/ \mathrm{cm}^{2}$. After $24 \mathrm{~h}$, the cells were transfected with $10 \mathrm{nM}$ Wasf2 siRNA or scramble siRNA (no. 4611; Ambion) complexed with Lipofectamine RNAiMAX (Invitrogen, Carlsbad, CA, USA). After the cells were cultured for a further 5 days, total RNA was extracted from the cells.

Statistical analysis. All experiments were repeated three to four times and representative results are shown. The data are presented as the mean \pm standard deviation, and were analyzed by the Student's t-test. Values of $\mathrm{P}<0.05$ were considered to indicate statistically significant results.

\section{Results and Discussion}

Regulation of gene expression by intermittent PTH administration. PTH has both anabolic and catabolic effects on bone depending on the mode of administration (11). Several molecules have previously been identified as mediators of the effects of intermittent PTH. In this study, we used the mouse osteoblastic cell line MC3T3-E1 to evaluate the anabolic effect of intermittent PTH administration using an in vitro model. The cells were cultured in the presence of PTH or in control medium for 1, 6 (intermittent PTH administration) or 48 h (continuous PTH administration) within each 48-h incubation cycle, and these cycles were carried out three times. To identify candidate intermittent PTH-responsive genes, we performed DNA microarray analyses to study the effect of intermittent PTH on gene expression. We used Agilent mouse DNA arrays that contain oligonucleotide probe sets representing 55,684 genetic elements, all the characterized mouse genes. Comparison of the intermittent (6 h) PTH1-34 treatment to the continuous PTH1-34 treatment revealed that $1 \%$ or less of the genes changed $>2$-fold (data not shown). Among these genes, the Wasf 2 gene was identified as a candidate intermittent PTH-responsive gene. To confirm this observation, RT-PCR and qRT-PCR analyses were performed to examine Wasf 2 mRNA expression levels in these cells. The expression level of Wasf 2 mRNA declined to $~ 80 \%$ following intermittent PTH1-34 (6 h) as compared with continuous PTH1-34 (48 h) (Fig. 1A and B).

PTH activates both the adenylate cyclase/PKA and the plasma membrane phospholipase C/PKC pathways and multiple mechanisms are involved in the anabolic effect induced by intermittent PTH treatment (12). Using PTH3-34 which lacks the PKA-activating domain, Wasf2 expression was also downregulated by intermittent treatment (1 and 6 h) (Fig. 1A and B). Treatment with intermittent PTH1-34 in combination with the PKC inhibitor, Go6976, which was 
A

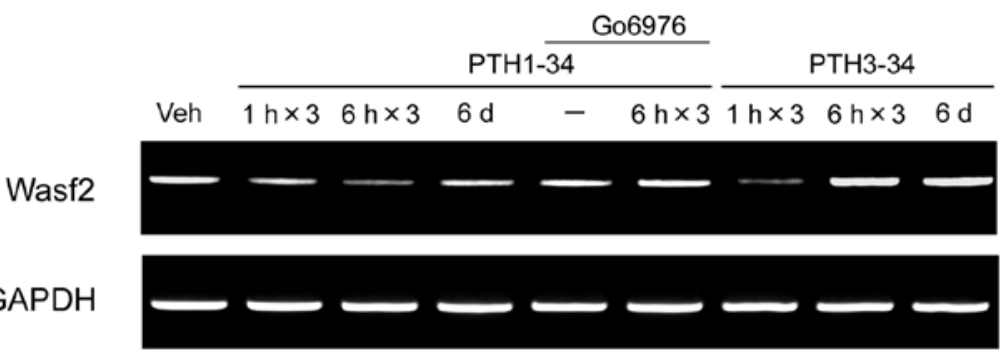

B

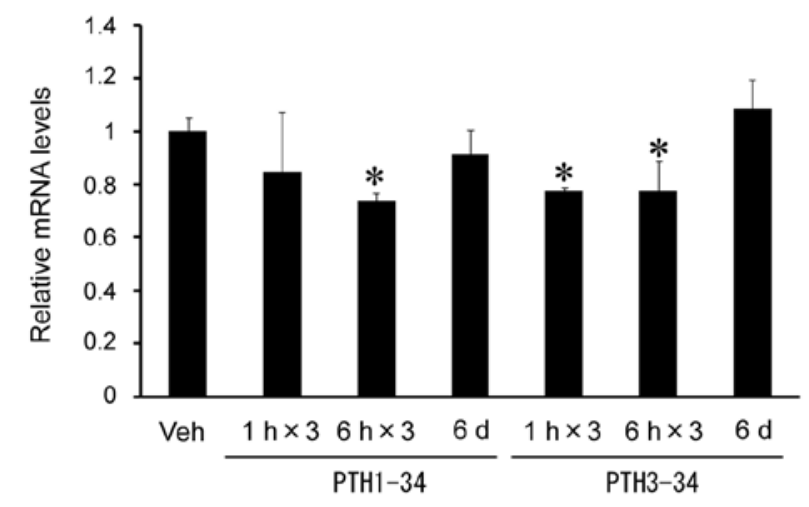

C

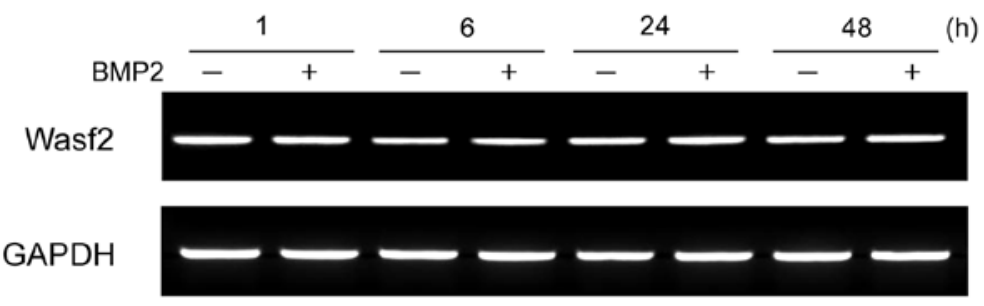

Figure 1. Regulation of Wasf2 mRNA expression by intermittent PTH administration in MC3T3-E1 cells. MC3T3-E1 cells were plated at $1 \times 10^{5}$ cells/ $/ \mathrm{cm}^{2}$. (A and B) After $24 \mathrm{~h}$, the cells were cultured in the presence of 10-8 M PTH1-34, 3-34 or vehicle with or without the PKC inhibitor Go6976 (100 nM) for $1(1 \mathrm{~h} \mathrm{x} 3), 6(6 \mathrm{~h} \mathrm{x} 3)$ or $48 \mathrm{~h}$ (6 days) within each 48-h incubation cycle. These cycles were carried out three times. Go6976 was added to cultures before treatment with PTH1-34. (C) MC3T3-E1 cells were cultured with BMP2 $(250 \mathrm{ng} / \mathrm{ml})$ for 1, 6, 24 and $48 \mathrm{~h}$. Total RNA was extracted from the cells, and mRNA expression of Wasf 2 was determined by reverse transcription-polymerase chain reaction (RT-PCR) (A). The level of Wasf 2 transcripts was evaluated by quantitative RT-PCR (qRT-PCR) (B and C). Data are shown as mean \pm SD. Each assay was performed on a separate experiment and was carried out in triplicate. "Significant difference, $\mathrm{P}<0.05$; t-test for paired data.

added to the culture before treatment, inhibited the downregulation of Wasf 2 mRNA expression. These results suggest that the response of Wasf 2 expression to intermittent PTH administration is mediated by the PKC signaling pathway. Since BMPs are known to regulate the differentiation and function of osteoblasts, we analyzed the level of Wasf 2 expression following administration of BMP2. However, BMP2 had no effect on Wasf2 mRNA expression (Fig. 1C).

Effects of the Wnt and BMP/Smad inhibitors on downregulation of Wasf 2 mRNA expression by intermittent PTH treatment. Previously, in vivo and in vitro studies have indicated that PTH-regulated anti-apoptotic signaling promotes osteoblastic differentiation and increases production and/or activation of osteogenic growth factors such as IGF-1 or BMP $(1,13)$. Recent studies in vitro have shown that Wnt signaling can mediate the actions of G-protein coupling receptors in many tissues (14-16) and have explored links between PTH and Wnt signaling pathways. Continuous PTH administration to MC3T3-E1 cells was found to increase the stabilized $\beta$-catenin level in those cells (17). PTH receptor signaling has also been shown to result in binding of the receptor to Lrp6, phosphorylation of Lrp6 and stabilization of $\beta$-catenin in osteoblasts (18). Most importantly, new data show that intermittent PTH administration in $\mathrm{Lrp5}^{-/-}$and $\mathrm{Lrp5}^{+/+}$mice show equal enhancement of skeletal mass (19), indicating that the anabolic effects due to PTH are independent of Lrp5 and thus of canonical Wnt signaling. Bergenstock and Partridge (20) provide evidence for a link between intermittent PTH actions and non-canonical Wnt signaling in bone. Despite all these observations, little is known about the contribution of Wnt signaling to the actions of intermittent PTH.

Recently, a novel class of small-molecule inhibitors that blocks Wnt signaling was identified (21). IWR-1 promotes $\beta$-catenin phosphorylation by stabilizing Axin-scaffolded destruction complexes; IWP-2 prevents palmitylation of Wnt proteins by porcupine, thereby blocking Wnt secretion and activity. Therefore, we examined the effects of these inhibitors on PTH administration. Although IWP-2 inhibited downregulation of Wasf 2 mRNA expression by intermittent PTH1-34 in MC3T3-E1 cells, IWR-1 did not inhibit its regulation (Fig. 2). The non-canonical Wnt pathway functions in a $\beta$-catenin-independent manner and signals are transduced 


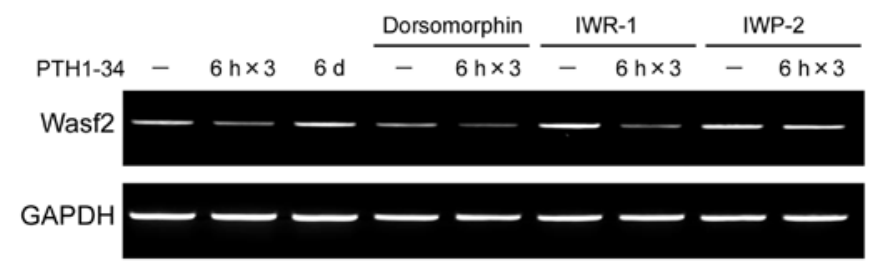

Figure 2. Effects of Wnt and BMP/Smad inhibitors on downregulation of Wasf2 mRNA expression by intermittent PTH treatment. MC3T3-E1 cells were plated at $1 \times 10^{5}$ cells $/ \mathrm{cm}^{2}$. After $24 \mathrm{~h}$, the cells were cultured without or with $10^{-8}$ M PTH1-34 for 6 (6 h x3) or $48 \mathrm{~h}$ (6 days) within each 48 -h incubation cycle. IWR-1 $(5 \mu \mathrm{M})$, IWP-2 $(0.5 \mu \mathrm{M})$ or dorsomorphin $(10 \mu \mathrm{M})$ was added to the culture before treatment with PTH1-34. These cycles were carried out three times. Total RNA was extracted from the cells, and then mRNA expression of Wasf 2 was determined by RT-PCR. Each assay was performed on a separate experiment and was carried out in triplicate.

through Fz family receptors and a co-receptor, such as Ror2 or RYK, but not Lrp-5/6 (22). Our data suggest that the Wasf2 response following intermittent PTH administration may mediate the non-canonical Wnt signaling pathway. It is also consistent with recent studies of PTH1R showing that Lrp6 is not required for intermittent PTH action. In contrast, the BMP/Smad inhibitor dorsomorphin was unable to regulate the Wasf2 response to PTH (Fig. 2), indicating that the $\mathrm{BMP} / \mathrm{Smad}$ signaling pathway could not mediate regulation of this gene by intermittent PTH, consistent with the results shown in Fig. 1C.

There are three types of pathways involved in noncanonical Wnt signaling. Among them, the $\mathrm{Wnt} / \mathrm{Ca}^{2+}$ pathway, in which non-canonical Wnts such as Wnt-5a bind to the Fz receptor and to a co-receptor such as Knypek or Ror2 and stimulate heterotrimeric $G$ proteins, increases intracellular calcium levels, decreases cyclin GMP (cGMP) levels, and then activates PKC or calcium/calmodulin-dependent protein kinase II (CamKII) to induce nuclear factor of activated T cells (NFAT) and other transcription factors (23). Takada et al (24) reported that the non-canonical Wnt pathway, through CamKII, transcriptionally induces Runx2 and represses peroxisome proliferator-activated receptor- $\gamma$ (PPAR- $\gamma$ ) transactivation. Taken together, these findings suggest that this pathway may be involved in the bone formation induced by intermittent PTH administration. Thus, the $\mathrm{Wnt} / \mathrm{Ca}^{2+}$ pathway induced through Wasf 2 expression may regulate cell adhesion and cell movement in bone tissue.

Effects of Wasf 2 knockdown on gene expression in MC3T3-E1 cells. Wasf 2 is ubiquitously expressed in mammalian cells, plays roles in lamellipodia formation and cell-cell contact organization under a Rho family small GTPase Rac1 signal and is the most mechanosensitive cytoskeleton-related gene (25). Based on the results of the present study, intermittent PTH administration regulates Wasf 2 expression. Until now, little was known of the molecular basis of action of Wasf 2 on osteoblasts. To investigate the function of Wasf2 in osteoblasts, we transfected MC3T3-E1 cells with Wasf2 siRNA. Transfection of Wasf2 siRNA reduced Wasf2 mRNA expression (Fig. 3A). In these cells, the expression levels of bone sialoprotein (BSP) and OPG mRNA were reduced by 20 and 60\%, respectively (Fig. 3B). Intermittent PTH1-34 admin-
A

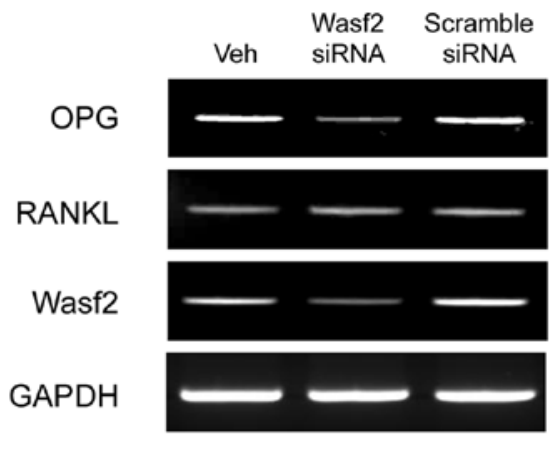

B
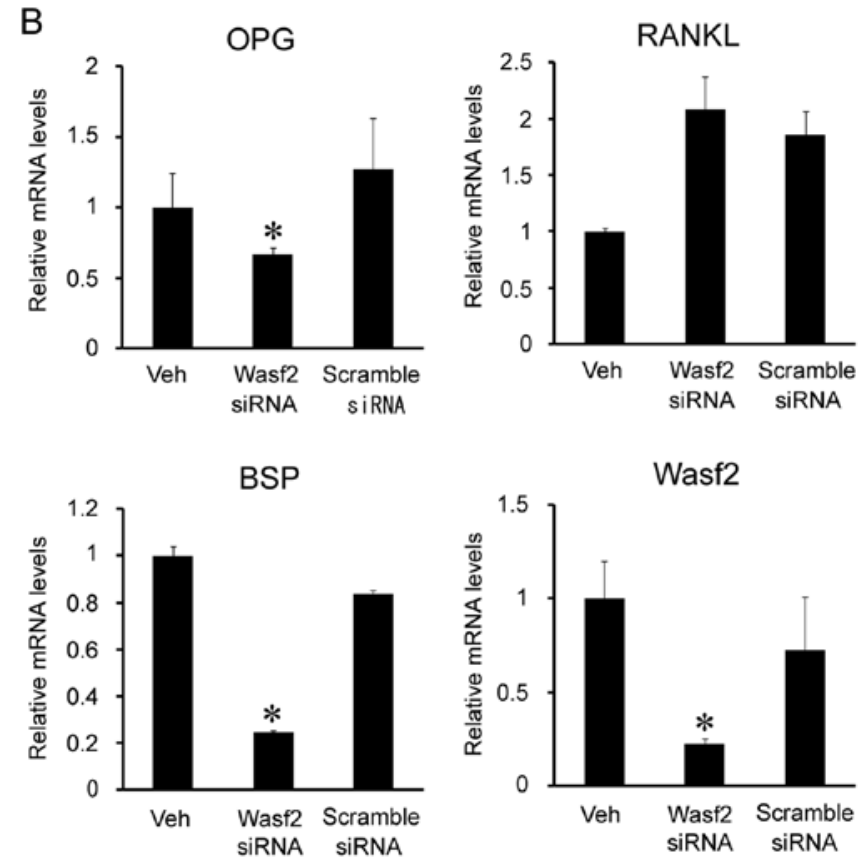

C

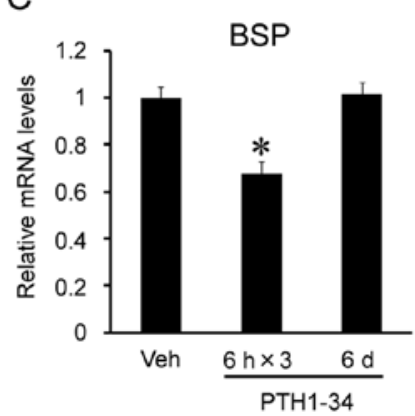

Figure 3. Effects of Wasf2 knockdown on gene expression in MC3T3-E1 cells. MC3T3-E1 cells were plated at $1 \times 10^{5}$ cells $/ \mathrm{cm}^{2}$. After $24 \mathrm{~h}$, cells were transfected with $10 \mathrm{nM}$ Wasf 2 siRNA or scramble siRNA complexed with Lipofectamine RNAiMAX. The cells were then cultured for a further 5 days. (A) Total RNA was extracted from the cells, and mRNA expression of OPG and RANKL was determined by RT-PCR. (B) The levels of OPG, RANKL and BSP transcripts were evaluated by qRT-PCR. (C) After $24 \mathrm{~h}$, the cells were cultured in the presence of $10^{-8} \mathrm{M}$ PTH1-34 or control medium for 6 ( $6 \mathrm{~h} \mathrm{x} 3)$ or $48 \mathrm{~h}$ (6 days) within each 48 -h incubation cycle. These cycles were carried out three times. The level of BSP transcripts was evaluated by qRT-PCR. Data are shown as mean \pm SD. Each assay was performed on a separate experiment and was carried out in triplicate. "Significant difference, $\mathrm{P}<0.05$; t-test for paired data

istration also reduced BSP expression (Fig. 3C), suggesting that downregulation of BSP expression by intermittent PTH may be mediated by Wasf 2 . 
BSP is a phosphorylated bone matrix glycoprotein and is an Arg-Gly-Asp (RGD)-containing protein which is mainly produced by mature osteoblasts and osteocytes. BSP is thought to function as an adaptor molecule between cells and bone mineral and may also be important in the differentiation, tissue organization and remodeling of bone (26). BSP is necessary for promoting cell adhesions through interaction with integrins $(27,28)$. In addition, the RGD motif in BSP can mediate both cell attachment and signaling activities (26). According to our experiments, cell adhesion and the cytoskeleton-related gene Wasf 2 could together regulate expression of BSP which may be involved in the regulation of cell attachment. It was previously reported that BSP also modulates the activity of osteoclasts through the vitronectin receptor which is present on the surfaces of osteoclasts (29), and it is also reported that the $\mathrm{Wnt} / \mathrm{Ca}^{2+}$ pathway can affect cell adhesion and cell movement during gastrulation $(30,31)$. The present study supports the hypothesis that intermittent PTH administration decreases Wasf 2 mRNA expression, and then regulates BSP expression by altering cell attachment and/or signaling activities, and thus regulates the osteoclastic activities in bone tissue.

\section{References}

1. Jilka RL: Molecular and cellular mechanisms of the anabolic effect of intermittent PTH. Bone 40: 1434-1446, 2007.

2. Ma YL, Cain RL, Halladay DL, et al: Catabolic effects of continuous human PTH (1-38) in vivo is associated with sustained stimulation of RANKL and inhibition of osteoprotegerin and gene-associated bone formation. Endocrinology 142 : 4047-4054, 2001.

3. Kronenberg HM, Lanske B, Kovacs CS, et al: Functional analysis of the PTH/PTHrP network of ligands and receptors. Recent Prog Horm Res 53: 283-303, 1998.

4. Jilka RL, Weinstein RS, Bellido T, Roberson P, Parfitt AM and Manolagas SC: Increased bone formation by prevention of osteoblast apoptosis with parathyroid hormone. J Clin Invest 104: 439-446, 1999.

5. Keller H and Kneissel M: SOST is a target gene for PTH in bone. Bone 37: 148-158, 2005.

6. Thrasher AJ and Burns SO: WASP: a key immunological multitasker. Nat Rev Immunol 10: 182-192, 2010.

7. Yamashita $H$, Ueda $K$ and Kioka N: WAVE2 forms a complex with PKA and is involved in PKA enhancement of membrane protrusions. J Biol Chem 286: 3907-3914, 2011.

8. Yamazaki D, Suetsugu S, Miki H, et al: WAVE2 is required for directed cell migration and cardiovascular development. Nature 424: 452-456, 2003.

9. Yan C, Martinez-Quiles N, Eden S, et al: WAVE2 deficiency reveals distinct roles in embryogenesis and Rac-mediated actinbased motility. EMBO J 22: 3602-3612, 2003.

10. Nakashima A, Katagiri T and Tamura M: Cross-talk between Wnt and bone morphogenetic protein 2 (BMP-2) signaling in differentiation pathway of C2C12 myoblasts. J Biol Chem 280 : 37660-37668, 2005.

11. Poole KE and Reeve J: Parathyroid hormone - a bone anabolic and catabolic agent. Curr Opin Pharmacol 5: 612-617, 2005.
12. Ishizuya $\mathrm{T}$, Yokose $\mathrm{S}$, Hori $\mathrm{M}$, et al: Parathyroid hormone exerts disparate effects on osteoblast differentiation depending on exposure time in rat osteoblastic cells. J Clin Invest 99: 2961-2970, 1997.

13. Zhang R, Edwards JR, Ko SY, et al: Transcriptional regulation of BMP2 expression by the PTH-CREB signaling pathway in osteoblasts. PLoS One 6: e20780, 2011.

14. Castellone MD, Teramoto H, Williams BO, Druey KM and Gutkind JS: Prostaglandin E2 promotes colon cancer cell growth through a Gs-axin-beta-catenin signaling axis. Science 310: 1504-1510, 2005.

15. Goessling W, North TE, Loewer S, et al: Genetic interaction of PGE2 and Wnt signaling regulates developmental specification of stem cells and regeneration. Cell 136: 1136-1147, 2009.

16. Liu Z and Habener JF: Glucagon-like peptide-1 activation of TCF7L2-dependent Wnt signaling enhances pancreatic beta cell proliferation. J Biol Chem 283: 8723-8735, 2008.

17. Tobimatsu T, Kaji H, Sowa $\mathrm{H}$, et al: Parathyroid hormone increases beta-catenin levels through $\mathrm{Smad} 3$ in mouse osteoblastic cells. Endocrinology. 147: 2583-2590, 2006.

18. Wan M, Yang C, Li J, et al: Parathyroid hormone signaling through low-density lipoprotein-related protein 6. Genes Dev 22: 2968-2979, 2008.

19. Sawakami K, Robling AG, Ai M, et al: The Wnt co-receptor LRP5 is essential for skeletal mechanotransduction but not for the anabolic bone response to parathyroid hormone treatment. J Biol Chem 281: 23698-23711, 2006.

20. Bergenstock MK and Partridge NC: Parathyroid hormone stimulation of noncanonical Wnt signaling in bone. Ann NY Acad Sci 1116: 354-359, 2007.

21. Chen B, Dodge ME, Tang W, et al: Small molecule-mediated disruption of Wnt-dependent signaling in tissue regeneration and cancer. Nat Chem Biol 5: 100-107, 2009.

22. Gordon MD and Nusse R: Wnt signaling: multiple pathways, multiple receptors, and multiple transcription factors. J Biol Chem 281: 22429-22433, 2006.

23. Wang HY and Malbon CC: Wnt-frizzled signaling to G-protein-coupled effectors. Cell Mol Life Sci 61: 69-75, 2004.

24. Takada I, Suzawa M, Matsumoto K and Kato S: Suppression of PPAR transactivation switches cell fate of bone marrow stem cells from adipocytes into osteoblasts. Ann NY Acad Sci 1116: 182-195, 2007.

25. Qian A, Di S, Gao X, et al: cDNA microarray reveals the alterations of cytoskeleton-related genes in osteoblast under high magneto-gravitational environment. Acta Biochim Biophys Sin 41: 561-577, 2009.

26. Ganss B, Kim RH and Sodek J: Bone sialoprotein. Crit Rev Oral Biol Med 10: 79-98, 1999.

27. Somerman MJ, Fisher LW, Foster RA and Sauk JJ: Human bone sialoprotein I and II enhance fibroblast attachment in vitro. Calcif Tissue Int 43: 50-53, 1988.

28. Helfrich MH, Nesbitt SA, Dorey EL and Horton MA: Rat osteoclasts adhere to a wide range of RGD (Arg-Gly-Asp) peptide-containing proteins, including the bone sialoproteins and fibronectin, via a beta 3 integrin. J Bone Miner Res 7: 335-343, 1992.

29. Lakkakorpi PT, Helfrich MH, Horton MA and Väänänen HK: Spatial organization of microfilaments and vitronectin receptor, alpha v beta 3 , in osteoclasts. A study using confocal laser scanning microscopy. J Cell Sci 104: 663-670, 1993.

30. Veeman MT, Axelrod JD and Moon RT: A second canon. Functions and mechanisms of beta-catenin-independent Wnt signaling. Dev Cell 5: 367-377, 2003.

31. Chisholm AD: Gastrulation: Wnts signal constriction. Curr Biol 16: R874-R876, 2006 